\title{
Characterization of TaSPP-5A gene associated with sucrose content in wheat (Triticum aestivum $\mathrm{L}$.)
}

Fanli Jing ${ }^{1,2}$, Yongping Miao ${ }^{1,2}$, Peipei Zhang ${ }^{1}$, Tao Chen ${ }^{1,2}$, Yuan Liu ${ }^{1,2}$, Jingfu Ma ${ }^{1,2}$, Mengfei Li ${ }^{1,2}$ and Delong Yang ${ }^{1,2^{*}}$

\begin{abstract}
Background: Sucrose, the major product of photosynthesis and the primary sugar transported as a soluble carbohydrate via the phloem, is a critical determinant for harvest yield in wheat crops. Sucrose-phosphatase (SPP) catalyzes the final step in the sucrose biosynthesis pathway, implying its essential role in the plant.

Result: In this study, wheat SPP homologs genes were isolated from chromosomes 5A, 5B, and 5D, designated as TaSPP-5A, TaSPP-5B, and TaSPP-5D, respectively. Sequence alignment showed one 1-bp Insertion-deletion (InDel) and three single nucleotide polymorphisms (SNPS) at TaSPP-5A coding region, forming two haplotypes, TaSPP-5Aa and TaSPP-5Ab, respectively. A derived cleaved amplified polymorphism sequence (dCAPS) marker, TaSPP-5A-dCAPS, was developed to discriminate allelic variation based on the polymorphism at position 1242 (C-T). A total of 158 varieties were used to perform a TaSPP-5A marker-trait association analysis, where two haplotypes were significantly associated with sucrose content in two environments and with thousand-grain weight (TGW) and grain length (GL) in three environments. Quantitative real-time PCR further revealed that TaSPP-5Aa showed relatively higher expression than TaSPP-5Ab in wheat seedling leaves, generally associating with increased sucrose content and TGW. The expression of TaSPP-5A and sucrose content in TaSPP-5Aa haplotypes were also higher than those in TaSPP-5Ab haplotypes under both 20\% PEG-6000 and 100 MM ABA treatment. Sequence alignment showed that the two TaSPP-5A haplotypes comprised 11 SNPs from -395 to -1962 bp at TaSPP-5A promoter locus, participating in the formation of several conserved sequences, may account for the high expression of TaSPP-5A in TaSPP-5Aa haplotypes. In addition, the distribution analysis of TaSPP-5A haplotypes revealed that TaSPP-5Aa was preferred in the natural wheat population, being strongly positively selected in breeding programs.

Conclusion: According to the SNPs detected in the TaSPP-5A sequence, two haplotypes, TaSPP-5Aa and TaSPP$5 A b$, were identified among wheat accessions, which potential value for sucrose content selection was validated by association analysis. Our results indicate that the favorable allelic variation TaSPP-5Aa should be valuable in enhancing grain yield by improving the sucrose content. Furthermore, a functional marker, TaSPP-5A-dCAPS, can be used for marker-assisted selection to improve grain weight in wheat and provides insights into the biological function of TaSPP-5A gene.
\end{abstract}

Keywords: Wheat, Thousand-grain weight, Sucrose content, TaSPP, Allelic variation, Functional marker

\footnotetext{
*Correspondence: yangdl@gsau.edu.cn

${ }^{2}$ College of Life Science and Technology, Gansu Agricultural University,

Lanzhou 730070, Gansu, China

Full list of author information is available at the end of the article
}

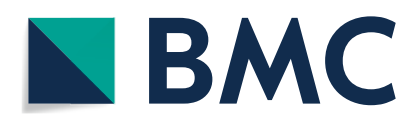

(c) The Author(s) 2022. Open Access This article is licensed under a Creative Commons Attribution 4.0 International License, which permits use, sharing, adaptation, distribution and reproduction in any medium or format, as long as you give appropriate credit to the original author(s) and the source, provide a link to the Creative Commons licence, and indicate if changes were made. The images or other third party material in this article are included in the article's Creative Commons licence, unless indicated otherwise in a credit line to the material. If material is not included in the article's Creative Commons licence and your intended use is not permitted by statutory regulation or exceeds the permitted use, you will need to obtain permission directly from the copyright holder. To view a copy of this licence, visit http://creativecommons.org/licenses/by/4.0/. The Creative Commons Public Domain Dedication waiver (http://creativeco $\mathrm{mmons}$.org/publicdomain/zero/1.0/) applies to the data made available in this article, unless otherwise stated in a credit line to the data. 


\section{Background}

Wheat (Triticum aestivum L.) is one of the most important cereal crops, and breeding new wheat varieties with higher grain production and stronger abiotic stress resistance is required to meet the projected requirements of wheat for an increasing world population. Due to the abundance and relative stability of single nucleotide polymorphisms (SNPs) in the genome, SNPs have been used as molecular markers to identify genes related with observed traits [1-3]. Many genes contributing to grain yield have been identified via SNPs genotyping in wheat, including sucrose synthase (TaSus2-2B), TaGW2, $T a G W 2-6 B$, cell wall invertase gene (TaCwi-A1), cytokinin oxidase/dehydrogenase (TaCKX6-D1), glutamine synthetase (TaGS1a), TaSAP1-A1, TaGASR7-A1, TaGS$D 1$, IAA-glucose hydrolase (TaTGW6), trehalose 6-phosphate phosphatase (TaTPP-6AL1) and sucrose-fructan 6-fructosyltransferase (6-SFT-A2) [1, 4-16].

In crop plants, sucrose serves a pivotal role in the distribution of carbon resources, and thus its accumulation and remobilization are critical determinants for harvest yield and grain quality [17]. Sucrose is synthesized in the cytosol by consecutive action of sucrose-phosphate synthase (SPS; EC 2.4.1.14) and sucrose phosphatase (SPP; EC 3.1.3.24) [18, 19]. SPS transfers the glucosyl moiety of UDP glucose onto fructose-6-phosphate to produce sucrose-6-phosphate, which is dephosphorylated by SPP to release free sucrose thereafter. The reaction catalyzed by SPP is essentially irreversible and displaces the reversible SPS reaction from equilibrium into the direction of net sucrose synthesis [18]. SPS has been generally recognized as a key regulator of the whole pathway of sucrose synthesis $[20,21]$, while the function of SPP in the regulation of assimilate partitioning has been relatively neglected [22]. Early reports showed that SPP activity appeared in large excess over SPS activity, indicating that SPP is unlikely to play a regulatory role in sucrose synthesis [23]. However, several pieces of evidence have revealed that SPP and SPS existed in a complex form in plants with remarkably similar maximum potential activities to each other [24-26]. SPP physically interacts with SPS, providing a new level of regulation of sucrose synthesis [27]. It suggests that SPP could have an essential role in metabolite channeling between the two enzymes and could more contribute to the control of sucrose biosynthesis than previously proposed $[22,26]$.

SPP belongs to a family of phosphatases/hydrolases with phosphohydrolase and C-terminal domains common in higher plants $[28,29]$ and is encoded by small gene families [30]. As so far, SPP genes have been cloned in different plant species, such as Arabidopsis thaliana, tomato (Lycopersicon esculentum), rice (Oryza sativa), maize (Zea mays), barley (Hordeum vulgare), and wheat
[31-33], where they constitute gene families with different number of members depending on the species. Four genomic SPP-like genes are available from Arabidopsis thaliana and rice, respectively, which have very similar exon-intron structures [31]. In wheat, three SPP-like cDNA (TaSPP1, TaSPP2, and TaSPP3) have been cloned with $94 \%$ identity at the nucleotide level with each other, indicative of homologous genes in wheat genomes [31]. The deduced amino acid sequences also show very high similarity with the barley $S P P 1$, maize $S P P 1$, and rice SPP1 and SPP2 sequences [31], suggesting that these are orthologous genes. However, due to the enormous genome size and allohexaploid nature of bread wheat, it is essential to ensure that primer sets for cloning genes are chromosome-specific [34]. Hitherto, the main objectives of this study were to characterize the genomic sequence of TaSPP, investigate the haplotype variation of TaSPP genes, and perform association analysis using multiple wheat varieties. Additionally, the geographic distribution and frequency of favorable allelic variation of TaSPP were analyzed. The purpose is to provide a new and effective molecular marker for breeding high yield wheat variety by marker-assisted selection.

\section{Results}

Cloning and nucleotide diversity in the region of TaSPP-5A

To obtain possible reference sequence of TaSPP genes, rice OsSPP (Os01g0376700) was used as the query against the EnsemblPlants (http://plants.ensemble.org/ index.html) database. Three SPP homologs with high similarity were obtained, named TaSPP1, TaSPP2, and TaSPP3 in a previous report [31]. The sequences of three homologous genes were used to perform sequence alignment with the genome sequences of three published wheat varieties, Chinese Spring, Fielder, and Zang 1817, which were accessed at the WheatOmics 1.0 website (http://202.194.139.32/blast/viroblast.php). Sequence alignment showed that the three homologous genes were distributed on $5 \mathrm{~A}, 5 \mathrm{~B}$, and $5 \mathrm{D}$ chromosomes, respectively. Therefore, they were renamed as TaSPP-5A, TaSPP-5B, and TaSPP-5D herein. The specific primer pairs of TaSPP-5A, TaSPP-5B, and TaSPP-5D genes were used to isolate the target genes in different wheat varieties (Additional file 1: Figure S1; Table S1). A 1-bp Insertion-deletion (InDel) and three single nucleotide polymorphisms (SNPs) at the coding region were identified in TaSPP-5A. The InDel and SNPs were located at 677, 1242, 1305, and $2077 \mathrm{bp}$, forming two haplotypes, TaSPP-5Aa and TaSPP-5Ab, respectively (Fig. 1). No polymorphisms were detected in TaSPP-5B and TaSPP-5D in the analysed sequence among the varieties included in this study. 


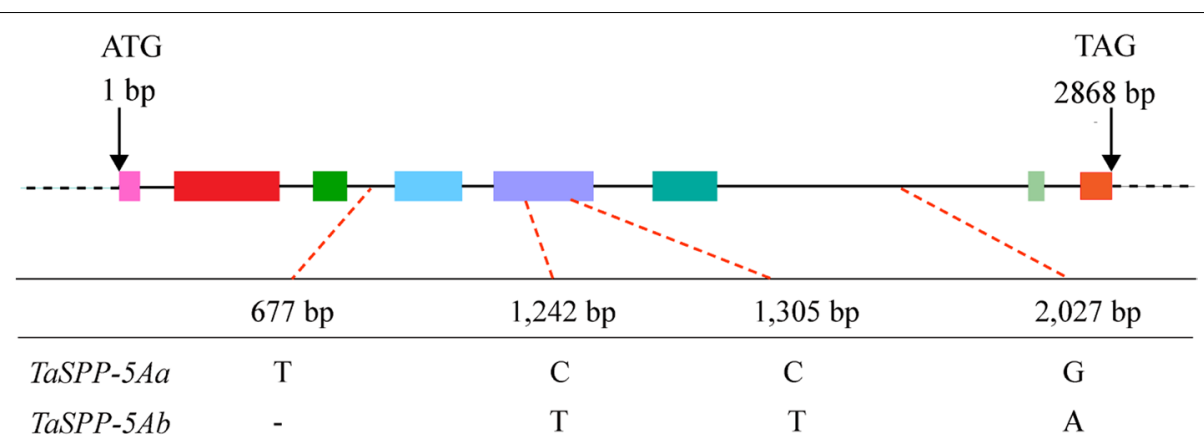

Fig. 1 Schematic diagram of TaSPP-5A structure, SNP and InDel in two haplotypes identified in TaSPP-5A among the wheat diversity panel. Exons were represented by different colored boxes, introns were denoted by blank lines, and UTRs were represented by a black dotted line

\section{Development of dCAPS markers in the TaSPP-5A gene}

Based on the sequence divergence of the allelic variants, a dCAPS marker was developed to distinguish the TaSPP-5A a and TaSPP-5Ab haplotypes (Fig. 2; Additional file 1: Table $\mathrm{S} 1$ ). The specific primers were designed to amplify an 843-bp fragment of TaSPP$5 A$ containing 1242 site $(\mathrm{C}-\mathrm{T})$. A primer pair, TaSPP5A-dCAPS-F and TaSPP-5A-dCAPS-R were used to amplify the 235-bp fragment spanning the variation site. The PCR product of 235-bp possessed allelic variation TaSPP-5Aa formed an EcoRI site GAATTC , whereas the corresponding site in $T a S P P-5 A b$ was GAATTT (Fig. 3a). Thus, the 235-bp PCR fragment of TaSPP-5Aa was digested into 20-bp and 215-bp parts by restriction enzyme EcoRI, whereas the corresponding product from TaSPP-5Ab remained 235-bp (Fig. 3b).

a

1230
$11-1$

TaSPP-5Aa AGAGACGTTGAGTTCCCTTACATCAAGGAG

TaSPP-5Ab AGAGACGTTGAGTTTCCTTACATCAAGGAG

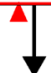

-G $\downarrow$ AATTC-

EcoRI

$\mathrm{b}$

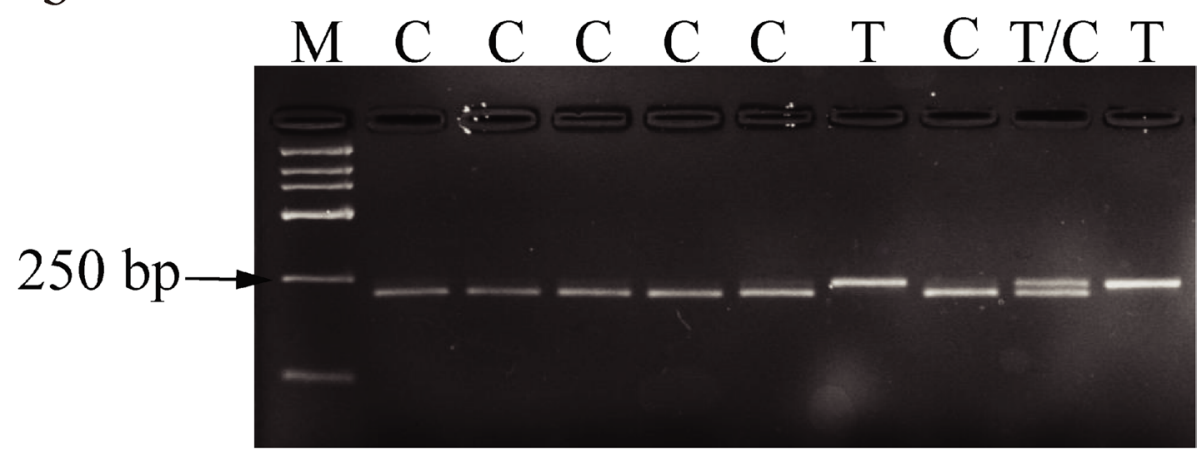

Fig. 2 Molecular marker of TaSPP-5A. (a) Molecular marker TaSPP-5A-dCAPS was developed based on the polymorphic SNP (C/T) site. The EcoRI restriction site and a base $\mathrm{G}$ mismatched to $\mathrm{A}$ are marked in red rectangle and red triangle, respectively (b) PCR products were digested by EcoRI. M is DL2000 DNA marker 

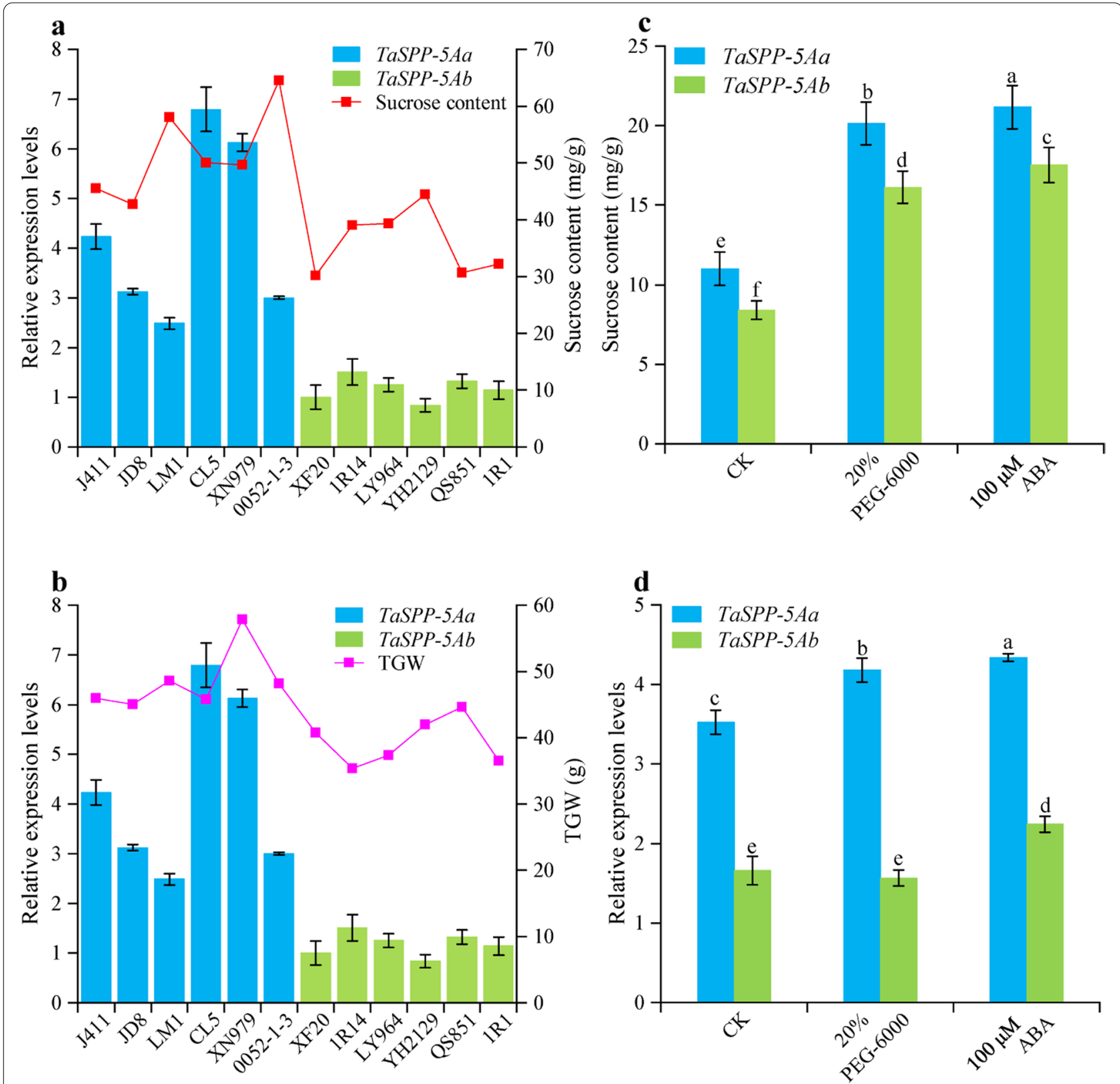

Fig. 3 The relative expression levels of TaSPP-5A in common wheat varieties carrying TaSPP-5Aa or TaSPP-5Ab haplotypes were analyzed by qRT-PCR. $(\mathbf{a}, \mathbf{b})$ Relationship between sucrose content $(\mathbf{a})$ and TGW (b) with TaSPP-5A expression in two TaSPP-5A haplotypes. The error bars represent SD from three biological replicates. Each replicate means the data got from a variety plant. XF20 was used as the reference sample. J411: Jing411; JD8: Jingdong8; LM1: Lumai1; CL5: Changle5; XN979: Xinong979; XF20: Xifeng20; LY964: Longyuan964; YH2129: Yunhan2129; QS851: Qingshan851. (c, d) Sucrose content (c) and expression level (d) in two haplotypes at seedling stage under the treatment of 20\% PEG-6000 and 100 $\mu \mathrm{M}$ ABA. The error bars represent SD from three biological replicates. Each replicate means the data got from a mixed pool which contained nine different variety plants. The ACTIN gene was used as an endogenous control in a, b, and d

\section{Association analysis between TaSPP-5A with sucrose content and grain-related traits}

To detect the effects of TaSPP-5A allelic variation, the average values of sucrose content, thousand-grain weight (TGW), and grain length (GL) between TaSPP-5A allelic variation were compared. A total of 158 varieties were used to validate the dCAPS marker for TaSPP-5A gene (Additional file 1: Table S2). A significant association between TaSPP-5A and sucrose content was observed in two environments (Table 1). The lines with allele TaSPP$5 A a$ had a significantly higher $(P<0.05)$ sucrose content than those with TaSPP-5Ab, suggesting a relationship 
Table 1 Association analysis of TaSPP-5A allelic variation and sucrose content in two environments

\begin{tabular}{llllll}
\hline Environment & Genotype & No. & $\begin{array}{l}\text { Sucrose content } \\
\text { Mean } \pm \text { SD }\end{array}$ & $\boldsymbol{F}$ & $\boldsymbol{P}$ value \\
\hline Yuzhong & TaSPP-5Aa & 115 & $19.98 \pm 1.10$ & 4.10 & $0.048^{*}$ \\
(2014-2015) & TaSPP-5Ab & 43 & $16.39 \pm 0.84$ & & \\
Tongwei & TaSPP-5Aa & 115 & $38.14 \pm 2.21$ & 4.53 & $0.038^{*}$ \\
$(2018-2019)$ & TaSPP-5Ab & 43 & $30.83 \pm 2.49$ & & \\
\hline
\end{tabular}

NO.: Number of accessions; ${ }^{*} P<0.05$ and ${ }^{* *} P<0.01$, respectively

$P$ values calculated by the $F$ statistics

between TaSPP-5A gene and sucrose content in wheat. The association analysis also showed that TaSPP-5Aa allelic variation had significantly higher $(P<0.05)$ TGW and GL than those with TaSPP-5Ab in three environments (Table 2). Thus, TaSPP-5Aa could be a favorable allelic variation for improving grain yield.

\section{TaSPP-5Aa haplotype had a higher expression level of TaSPP-5A gene compared with TaSPP-5Ab}

To analyze the effect of favorable allelic variation, 12 varieties possessing TaSPP-5Aa or TaSPP-5Ab were from 158 varieties were used to test the expression level of TaSPP-5A gene. qRT-PCR showed that genotypes with TaSPP-5Aa had relatively higher expression levels than those with $T a S P P-5 A b$ in grains at the grain-filling stage (Fig. 3a and b), as well as in 14-day-old seedlings (Additional file 1: Figure S3). Consistently, the sucrose content and TGW were generally higher in TaSPP-5Aa haplotype than those in TaSPP-5Ab (Fig. $3 \mathrm{a}$ and $\mathrm{b}$ ), suggesting that the expression level of TaSPP-5Aa might help to maintain high sucrose content and increase wheat yield.

As the metabolism of sucrose has been shown to involve in response to abiotic stress [35, 36], to verify whether TaSPP-5A serves any specific roles in the metabolism of sucrose under abiotic stress conditions. Nine wheat varieties with haplotype TaSPP-5Aa and nine wheat varieties with haplotype $T a S P P-5 A b$ were used to establish two mixed pools under different abiotic stress treatments. Although the sucrose content was significantly induced $(P<0.05)$ under both $20 \%$ PEG-6000 and $100 \mu \mathrm{M} \mathrm{ABA}$ treatment at two haplotypes (Fig 3c), the expression level of TaSPP-5A was slightly or barely induced at haplotypes TaSPP-5Aa or haplotypes TaSPP$5 A b$, respectively (Fig $3 \mathrm{~d}$ ), indicating TaSPP-5A may not play a key role under abiotic stress.

\section{Two TaSPP-5A haplotypes comprised 11 SNPs} at the promoter region of TaSPP-5A, generating putative transcription factor binding sites

As TaSPP-5Aa haplotype had higher expression of TaSPP-5A gene than TaSPP-5Ab, we further detected polymorphisms in the 2,000-bp DNA fragment of TaSPP$5 A$ promoter by using 640 varieties from the WheatUnion website (http://wheat.cau.edu.cn/WheatUnion/) and 21 varieties from 158 varieties used for association analysis. A total of 11 SNPs detected in the promoter of TaSPP-5A were specifically existed in haplotype TaSPP$5 A$ or haplotype TaSPP-5Ab, at $-395,-726,-830,-1057$, $-1335,-1554,-1558,-1741,-1796,-1831$, and -1962 bp positions, respectively, upstream of the start codon ATG (Fig. 4). Bioinformatic analysis showed that these 11 SNPs participate in the formation of several transcription factor binding sites, such as MYB and B3 binding site (Fig. 4; Additional file 1: Table S3). Therefore, the transcription factor binding site specifically contained in the promoter of TaSPP-5A gene in TaSPP-5Aa haplotypes may account for the high expression of TaSPP-5A.

TaSPP-5Aa underwent selection in Chinese wheat breeding Artificial selection leaves strong footprints in genomes. As TaSPP-5Aa allelic variation had high expression level of TaSPP-5A gene, as well as high TGW and GL, we wondered whether the TaSPP-5Aa haplotypes were positively selected. Then, the geographic distribution of two TaSPP$5 A$ haplotypes was evaluated using 252 cultivated accessions from nine provinces in China (Fig. 5a; Additional

Table 2 Association analysis of TaSPP-5A allelic variation and yield-related traits in three environments

\begin{tabular}{|c|c|c|c|c|c|c|c|c|}
\hline Environments & Genotype & No. & $\begin{array}{l}\text { TGW } \\
\text { Mean } \pm \text { SD }\end{array}$ & $F$ & $P$ value & $\begin{array}{l}\mathrm{GL} \\
\text { Mean } \pm \mathrm{SD}\end{array}$ & $F$ & $P$ value \\
\hline \multirow{2}{*}{$\begin{array}{l}\text { Tongwei } \\
\text { (2017-2018) }\end{array}$} & TaSPP-5Aa & 115 & $49.70 \pm 0.50$ & \multirow[t]{2}{*}{8.53} & \multirow[t]{2}{*}{$0.04^{*}$} & $6.92 \pm 0.04$ & \multirow[t]{2}{*}{4.10} & \multirow[t]{2}{*}{$0.045^{*}$} \\
\hline & $T a S P P-5 A b$ & 43 & $46.69 \pm 0.93$ & & & $6.73 \pm 0.08$ & & \\
\hline \multirow{2}{*}{$\begin{array}{l}\text { Tongwei } \\
\text { (2018-2019) }\end{array}$} & TaSPP-5Aa & 115 & $42.87 \pm 0.82$ & \multirow[t]{2}{*}{3.96} & \multirow[t]{2}{*}{$0.049^{*}$} & $6.92 \pm 0.04$ & \multirow[t]{2}{*}{5.32} & \multirow[t]{2}{*}{$0.023^{*}$} \\
\hline & $T a S P P-5 A b$ & 43 & $39.56 \pm 1.14$ & & & $6.75 \pm 0.05$ & & \\
\hline \multirow{2}{*}{$\begin{array}{l}\text { Tongwei } \\
\text { (2019-2020) }\end{array}$} & TaSPP-5Aa & 115 & $46.22 \pm 0.42$ & \multirow[t]{2}{*}{11} & \multirow[t]{2}{*}{$0.001^{* *}$} & $5.98 \pm 0.04$ & \multirow[t]{2}{*}{4.11} & \multirow[t]{2}{*}{$0.045^{*}$} \\
\hline & $T a S P P-5 A b$ & 43 & $43.36 \pm 0.79$ & & & $5.82 \pm 0.05$ & & \\
\hline
\end{tabular}

NO.: Number of accessions; TGW, thousand-grain weight; GL, Grain length; ${ }^{*} P<0.05$ and ${ }^{*} P<0.01$, respectively

$P$ values calculated by the $F$ statistics 


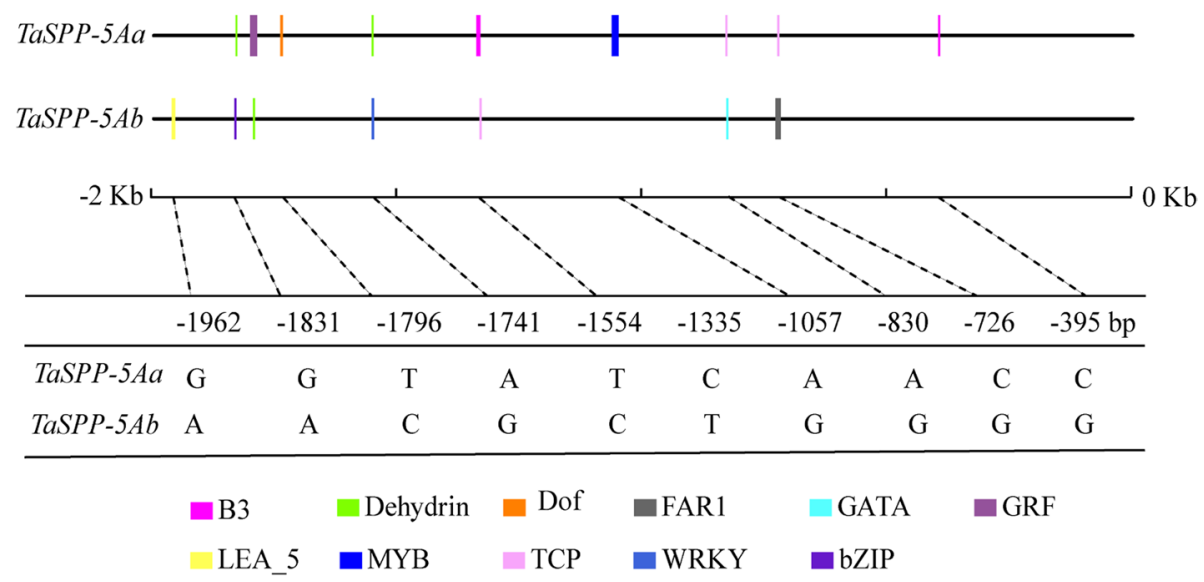

Fig. 4 The distribution of main cis-regulatory elements contained SNPs site in the promoter region of the two haplotypes of TaSPP-5A gene

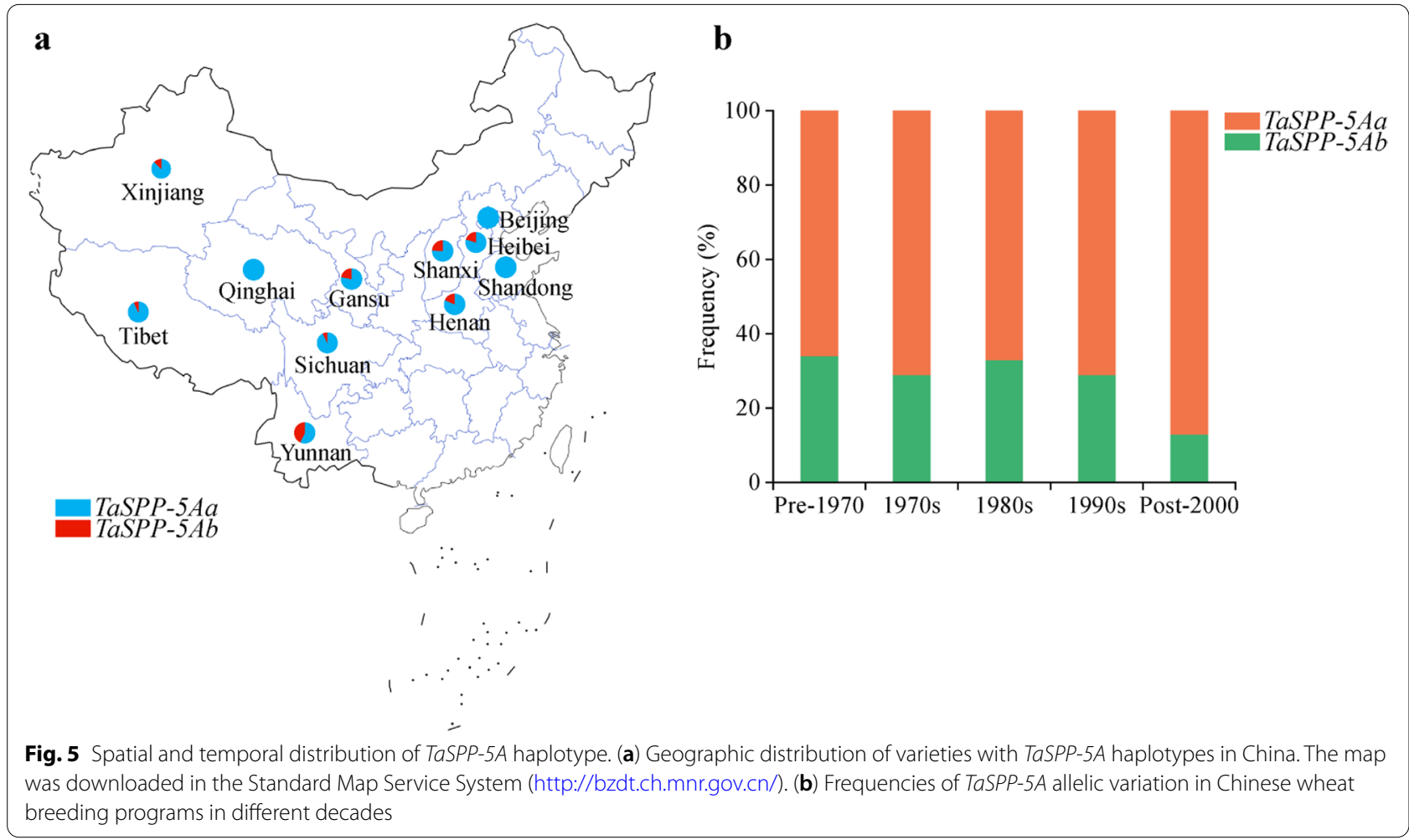

file 1: Table S4). The TaSPP-5Aa haplotype frequencies from 11 provinces in China were 78\% (Gansu), 100\% (Beijing), 81.8\% (Henan), 80\% (Hebei), 100\% (Qinghai), 100\% (Shandong), 75.7\% (Shanxi), 92.9\% (Sichuan), 93.2\% (Tibet), 87.5\% (Xinjiang), and 57.1\% (Yunnan). Geographic distribution patterns demonstrated that a favorable TaSPP-5Aa haplotype was strongly selected.

To further investigate whether the TaSPP-5Aa haplotypes were positively selected during the course of
Chinese wheat breeding, the frequencies of TaSPP-5A haplotypes in the historical population of 153 accessions were detected in 10-year intervals (pre-1970, 1970s, 1980s, 1990s, and post-2000) (Fig. 5b; Additional file 1: Table S5). The results revealed that TaSPP-5Aa underwent positive selection, while TaSPP-5A $b$ underwent negative selection, during Chinese wheat breeding process. 


\section{Discussions}

\section{Putative mechanism of TaSPP-5A gene in sucrose content}

As a product of photosynthesis, sucrose is the main form of carbohydrate translocated from the source to sinks such as seeds and roots [30]. The SPP encoding genes have been described in different plant species such as Arabidopsis, tomato, rice, wheat, maize, and coffee [24, 31]. Previous studies have proved that the change of SPP structure can improve the catalytic efficiency and sucrose content [37], and the decreased level of SPP in transgenic tobacco inhibited photosynthesis and altered carbohydrate partitioning with reduced growth rate [22]. The SPP activity repressed in transgenic potato tubers (Solanum tuberosum) also results in the decrease of sucrose content [33]. Therefore, SPP gene plays a vital role in the regulation of sucrose synthesis. In this study, a 1-bp InDel and three SNPs in the coding region and 11 SNPs in the promoter region of TaSPP-5A were identified, exhibiting a significant association with sucrose content.

Sucrose serves as an energy and signaling molecule required for tissue metabolism and as an osmotic to prevent tissue damage in response to abiotic stress [35, 38, 39]. Previous studies have proved that drought stresses increased the sucrose content in leaf and root tissues and phloem sap of rice indica varieties [40]. Several sucrose synthesis- and transport-related genes have been reported to be involved in response to abiotic stress $[35,36,41]$. Sucrose transporters (SUTs) play important roles in the sugar partitioning of source and sink organs. In Arabidopsis, the expression of AtSUC2 and AtSUC4 was significantly induced under salt, osmotic, low temperature, and exogenous abscisic acid (ABA) treatments, and the loss-of-function mutation of AtSUC2 and AtSUC4 displayed sensitive phenotype responses to abiotic stress and ABA treatment [41]. In Sweet potato, IbSUT4 also served as a positive regulator in plant stress tolerance through ABA signaling pathway [35]. Sucrose is synthesized by consecutive action of SPS and SPP. The activity of SPS was significantly higher in seedlings of drought-tolerant wheat varieties under normal growth conditions than non-drought-tolerant varieties [36]. The activity of SPS in drought-tolerant wheat varieties was also significantly increased after drought stress treatment [36]. Whether SPP genes participate in the regulation of plant abiotic stress tolerance was still unclear. In this study, the average sucrose content was significantly higher in TaSPP-5Aa haplotype than those in TaSPP$5 A b$ under both 20\% PEG-6000 and $100 \mu \mathrm{M}$ ABA treatments (Fig. 3d), the rainfall at Tongwei farm station is lower than that at Yuzhong farm station (Supplemental files Fig S4), we speculate that appropriate abiotic is one of the factors that affect the increase in sucrose content, but the expression level of TaSPP-5A was slightly or barely induced at haplotypes $T a S P P-5 A a$ or haplotypes TaSPP-5Ab, respectively. Therefore, these results implied that the TaSPP-5A may not play a key role under abiotic stress. Previous researches have shown that the developmental traits were more likely to be controlled by the genes at the A genome, whereas adaptive traits, such as responses to stress and disease, appeared more likely to be controlled by the genes at B and D genomes [42-44]. Therefore, we hypothesized TaSPP-5A may mainly serve pivotal roles in the development of wheat grain.

\section{The transcript regulation of TaSPP-5A gene}

Transcriptional regulation plays pivotal roles in the activation and suppression of gene expression, and is primarily controlled through gene promoters and their contributing cis-regulatory elements [45]. The expression level of TaSPP-5A gene was higher in TaSPP-5Aa haplotypes than that in TaSPP-5Ab haplotypes, we assumed that the SNPs in TaSPP-5A promoter region might affect the expression of TaSPP-5A, thus resulting in sucrose content variation in wheat. We used WheatUnion to investigate the diversity of TaSPP-5A promoter in 600 wheat varieties, and 11 SNPs were found in the promoter region of TaSPP-5A. Analysis of promoter cis-regulatory elements showed that the TaSPP-5Aa haplotypes specifically contained several transcription factor binding sites in the promoter region of TaSPP-5A gene, such as MYB and B3 transcription factor binding sites, which were not observed in TaSPP-5Ab haplotypes (Fig. 5). Previous studies have shown that MYB-type TFs participated in regulating sugar metabolism and the response of multiple abiotic stress. TaMYB13, an R2R3-MYB TF, has been characterized as a transcriptional activator of the fructan synthesis pathway in wheat [46]. CiMYB3 and CiMYB5 selectively bound the promoter of 1-FEH1, 1-FEH2a, and $1-F E H 2 b$ genes to enhance these genes' expression, without affecting the expression of the fructosyltransferase gene [47]. In potato, MYB TF positively regulated the expression of sucrose hydrolase genes SUSY1 and INV2 by directly binding to their promoters [48]. B3, the AFL (ABI3/FUS3/LEC2) subfamily of the B3 TFs, not only alter hormone biosynthesis, mainly involved in ABA and gibberellin, but also regulate the expression of other TFs and/or regulate their downstream activities through protein-protein interactions [49]. Therefore, we assumed that some transcription factors, such as MYB or B3, may specifically bind with the promoter of TaSPP-5Aa, but not TaSPP-5Ab, and promote the expression of TaSPP$5 A a$, thus resulting in high content of sucrose in wheat.

\section{The functional marker TaSPP-5A-dCAPS}

Functional marker derived from polymorphic sites within genes conferring specific phenotypes has been 
widely used for marker-assisted selection in wheat breeding, providing a strategy for accelerating the breeding process $[50,51]$. Sucrose accumulation- and remobilization-related traits have attracted the attention of breeders for many years, and are generally accepted as determiners of wheat yield. According to the SNPs detected in the TaSPP-5A sequence, two haplotypes, TaSPP-5Aa and $T a S P P-5 A b$, were identified among wheat accessions. Therefore, we designed a genome-specific primer set to separate orthologous genomic sequences among different wheat varieties and developed a dCAPS marker. Subsequently, their potential value for sucrose content selection was validated by association analysis. In our study, the dCAPS marker TaSPP-5A-dCAPS developed from TaSPP-5A was associated with sucrose content in flag leaf at the grain-filling stage (Table 1), and we also found that genotypes with favorable allelic variation TaSPP-5Aa had higher TGW and GL than TaSPP-5Ab allelic variation (Table 2). The functional marker TaSPP-5A-dCAPS can be used for marker-assisted selection to improve grain yield, and also for providing a theoretical basis for later research on sucrose accumulation and remobilization in wheat.

\section{Conclusions}

The TaSPP-5A gene was cloned. One 1-bp InDel and three SNPs were observed. A molecular marker TaSPP5A-dCAPS was developed based on an SNP at 1242bp (T-C). Association analysis revealed that favorable allelic variation TaSPP-5Aa (C) was associated with high sucrose content, TGW, and GL, compared with TaSPP$5 A b$ allelic variation. qRT-PCR showed the expression of TaSPP-5A gene in nine haplotypes, exhibiting a significantly higher expression in TaSPP-5Aa than in TaSPP$5 A b$. The frequency of favorable allelic variation also found that allelic variation TaSPP-5Aa underwent strong selection in wheat breeding. Our results provide a functional marker, TaSPP-5A-dCAPS, which can be used for marker-assisted selection to improve grain weight in wheat, and also provides insights into the molecular regulation of TaSPP-5A gene.

\section{Materials and methods Plant materials}

Varieties, including Longxuan987, Xifeng20, Lumai15, and $1 \mathrm{R} 1$ with significantly different sucrose contents, were used to clone the full-length sequences of TaSPP$5 A$ and identify allelic variations. A total of 158 wheat varieties was used for association analysis of polymorphic markers with agronomic traits (Additional file 1: Table S2), which was provided by Dr. Ruilian Jing at Chinese Academy of Agricultural Sciences [52]. The geographic distribution of two TaSPP-5A haplotypes was evaluated using 252 varieties, including 190 varieties accessed from WheatUnion and 62 varieties from 158 varieties used for association analysis (Additional file 1: Table S4). The frequencies of TaSPP-5A haplotypes were detected in the historical population of 153 varieties, including 126 varieties accessed from WheatUnion and 27 varieties from 158 varieties used for association analysis (Additional file 1: Table S5). A total of 28 materials were both used for geographic distribution and frequencies analysis. Nine wheat varieties with haplotype TaSPP$5 A a$ and nine wheat varieties with haplotype TaSPP-5Ab were used to establish two mixed pools under per stress treatment. Three biological repeats were performed to analyze the data. The seeds were cultivated in a glasshouse at $16 / 8 \mathrm{~h}\left(\right.$ light/dark, $\left.25 / 23^{\circ} \mathrm{C}\right)$. The varieties were

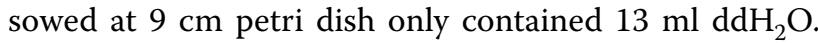
The water was poured out after 14 days of growing, and the seedlings were rewatered with $25 \mathrm{ml} 100 \mu \mathrm{M}$ abscisic acid (ABA) and 20\% polyethylene glycol-6000 (PEG6000 ), then the leaves of seedlings were removed after five days of treatment for the determination of sucrose content and the expression level of TaSPP-5A. All wheat accessions were legally obtained from Chinese Crop Germplasm Resources Information System (http://www. cgris.net/zhongzhidinggou/index.php).

\section{Field trials}

The natural population was sown at Yuzhong farm station, Gansu, China $\left(35^{\circ} 48^{\prime} \mathrm{N}, 104^{\circ} 18^{\prime} \mathrm{E}, 1860 \mathrm{~m}\right.$ ASL) in 2014-2015, and at Tongwei farm Station, Gansu, China $\left(35^{\circ} 110^{\prime} \mathrm{N}, 105^{\circ} 190^{\prime} \mathrm{E}, 1740 \mathrm{~m} \mathrm{ASL}\right)$ in $2017-2018,2018-$ 2019, and 2019-2020. Sucrose content was measured under two environments (2014-2015 and 2018-2019), and TGW and GL were calculated under three environments (2017-2018, 2018-2019, and 2019-2020). Field experimental designs under each environment were randomized complete blocks with three repeats for each variety. Each repeat means the data got from a variety plant. Each plot contained $1 \mathrm{~m}$ long with six rows spaced $20 \mathrm{~cm}$ apart. Nutrition supplied to all treatments was nitrogen $(\mathrm{N})$ of $180 \mathrm{~kg} \mathrm{hm}^{-2}$, phosphorus $\left(\mathrm{P}_{2} \mathrm{O}_{5}\right)$ of 150 $\mathrm{kg} \mathrm{hm}^{-2}$, and potassium $\left(\mathrm{K}_{2} \mathrm{O}\right)$ of $60 \mathrm{~kg} \mathrm{hm}^{-2}$ only at sowing. Other aspects of field management followed the local practices in wheat production. All the fresh samples were first dried at $100{ }^{\circ} \mathrm{C}$ for $30 \mathrm{~min}$ and thereafter dried at 80 ${ }^{\circ} \mathrm{C}$ until constant dry weight. The dehydrated samples were chipped into pieces of 3-5 $\mathrm{mm}$ in length. Subsequently sucrose content was measured using the Resorcin method as described by Yemm et al. [53]. Using a Microplate Reader scanned wavelengths at $500 \mathrm{~nm}$ reflectance mode. Agronomic traits have been measured by the SC-G2 kernel testing equipment developed by Wanshen Science and Technology Ltd. (Hangzhou, China) 


\section{Cloning and sequence analysis of TaSPP-5A}

The rice gene OsSPP (GenBank accession: AA752973) was used as the query for BLAST search against the EnsemblPlant (http://plants.ensembl.org/index.html). Based on the sequence alignment, a specific primer pair of TaSPP-5A-F1/R1 was designed to amplify wheat $S P P$ $5 A$ gene (Additional file 1: Table S1). Genomic DNA from fresh leaves of 14-day-old wheat seedlings was extracted by CTAB method [54]. PCR was performed in a total volume of $20 \mu \mathrm{L}$, including $2 \mathrm{pM}$ of each primer, 1.6 $\mu \mathrm{L}$ of $2.5 \mathrm{mM}$ dNTPs, $150 \mathrm{ng}$ of template DNA, $4 \mu \mathrm{L}$ of $5 \times$ TransStart FastPfu buffer, and $0.4 \mu \mathrm{L}$ of $2.5 \mathrm{U}$ TransStart FastPfu DNA Polymerase (TransGen Biotech, Beijing). The PCR conditions were an initial denaturation at $95^{\circ} \mathrm{C}$ for $5 \mathrm{~min}$, followed by 35 cycles of $95^{\circ} \mathrm{C}$ for 1 min, annealing $57^{\circ} \mathrm{C}$ for $20 \mathrm{~s}$, and extension at $72^{\circ} \mathrm{C}$ for $1.5 \mathrm{~min}$, with a final extension of $72^{\circ} \mathrm{C}$ for $10 \mathrm{~min}$. The PCR products were separated by $1 \%$ agarose gels electrophoresis and the target bands recovered, cloned into the pEASY-Blunt Cloning vector (TransGen Biotech, Beijing), and sequenced by Shanghai Sangon Biotech Co. Ltd (http://www.sangon.com). The genomic DNA sequences of the TaSPP-5A were aligned using the SeqMAN and MegAlign software (DNASTAR Lasergene 6.1.0) to detect allelic variants among accessions.

\section{Polymorphism identification and development of a functional marker}

Based on the SNP polymorphism sites (1242 bp-C/T), the specific primers (Additional file 1: Table S1) were designed to amplify TaSPP and a dCAPS was developed. By two rounds of PCR, the sequence of TaSPP-5A at 1242 sites (C-T) was amplified by TaSPP-5A-F2/R2, then the first round PCR products were diluted 200 times, and the second round PCR was performed with the primer pair TaSPP-5A-dCAPS-F/R using $1 \mu \mathrm{L}$ diluted PCR products as template. Finally, PCR products were digested with the restriction enzyme EcoRI (Sangon Biotech, Shanghai) and the digested PCR products were separated using $5 \%$ agarose gels.

\section{RNA extraction and quantitative Real-Time PCR}

Total RNA samples were extracted using an E.Z.N.A. ${ }^{\circledR}$ Plant RNA Kit (Omega, Shanghai). First-strand cDNA was synthesized with Rever Tra Ace qPCR RT Master Mix with gDNA Remover (TOYOBO, Japan). Quantitative real-time PCR (qRT-PCR) was performed for gene expression studies. Primer sequences used to amplify TaSPP-5A and TaACTIN (control) genes were shown in Table S1. qPT-PCR was performed with Roche LightCycler ${ }^{\circledR} 96$ (Roche, Switzerland) using the KOD SYBR qPCR Mix (TOYOBO, Japan). The reaction procedure was as follows: thermal cycling at $98^{\circ} \mathrm{C}$ for 120 $\mathrm{s}$, followed by 40 cycles at $98^{\circ} \mathrm{C}$ for $10 \mathrm{~s}, 60^{\circ} \mathrm{C}$ for $10 \mathrm{~s}$, and $68^{\circ} \mathrm{C}$ for $30 \mathrm{~s}$. Three biological repeats were used for qRT-PCR analysis. Each repeat means the data got from a variety plant. The relative transcript level of TaSPP-5Aa and TaSPP-5Ab was determined using the $2^{-\triangle \Delta \mathrm{Ct}}$ method [55].

\section{Statistical analysis}

One-way analysis of variance (ANOVA) was used to determine the significance between different haplotypes and phenotypic traits in SPSS19.0 software ( http://www. ibm.com ).

\section{Analysis of the cis-regulatory elements in the promoter}

The sequences of the 2,000-bp promoter in TaSPP-5A genes were retrieved from the EnsemblPlants database (http://plants.ensembl.org/index.html). Wheat genome variation consortium database (http://wheat.cau.edu. $\mathrm{cn} /$ WheatUnion/) was applied to obtain variant loci in promoters of different materials [56-58]. PlantPAN 3.0 (http://PlantPAN.itps.ncku.edu.tw/) was used to analyze the cis-regulatory elements in the promoter of TaSPP$5 A$. The distribution of identified cis-regulatory elements was drawn by the GSDS (http://gsds.gao-lab.org/).

\section{Abbreviations}

SPP: Sucrose phosphatase; dCAPS: Derived cleaved amplified polymorphic sequence; SNP: Single nucleotide polymorphism; Indel: Insertion and Deletion; TGW: Thousand-grain weight; GL: Grain length.

\section{Supplementary Information}

The online version contains supplementary material available at https://doi. org/10.1186/s12870-022-03442-x.

Additional file 1: Figure S1. The PCR amplification of TaSPP-5A gene in different wheat varieties. Figure S2. Alignment of the cloned TaSPP-5A orthologs. The restriction site ECORI and the locations of primers TaSPP5A-F2/R2 and TaSPP-5A-dCAPS-F/R were labeled by lines with arrow. Figure S3. The expression level of TaSPP-5A and the content of sucrose in 14-day-old wheat seedlings carrying TaSPP-5Aa or TaSPP-5Ab haplotypes. Figure S4. The rainfall for each growing season in the two tested environments. Table S1. Primer sequences used in this study. Table S2. The information of the wheat diversity panel and their genotypes of TaSPP-5A alleles. Table S3. Cis-regulatory elements contained SNPS site in the promoter region of the two haplotypes of TaSPP-5A gene and their sequences. Table S4. The information of the wheat diversity panel and geographic distribution of TaSPP-5A alleles. Table S5. The information of the wheat diversity panel and their genotypes of TaSPP-5A alleles in the different decades

\section{Acknowledgements}

We thank Dr. Ruanli Jing at Chinese Academy of Agricultural Sciences for providing the nature population (158 wheat varieties).

\section{Authors' contributions}

$\mathrm{DLY}, \mathrm{TC}$, and PPZ designed the study. FLJ, YPM, JFM, MFL, and PPZ performed most of the experiments. FLJ and YPM performed data analysis. FLJ wrote the 
manuscript. DLY reviewed and edited the manuscript. All authors read and approved the final manuscript.

\section{Funding}

This work was financially supported by the Research Program Sponsored by Gansu Provincial Key Laboratory of Aridland Crop Science, Gansu Agricultural University, China (GHSJ 2020-Z4), the National Natural Science Foundation of China (31760385), and the Key Research and Development Program of Gansu Province, China (21YF5NA089).

\section{Availability of data and materials}

All data generated or analyzed during this study are included in this published article and its supplementary information files. The datasets used and/or analyzed during the current study are available from the corresponding author on reasonable request. The sequencing data has been submitted to the NCBI SRA database (BioProject ID PRJNA762528, https://www.ncbi.nlm.nih.gov/sra/ PRJNA762528).

\section{Declarations}

Ethics approval and consent to participate

Not applicable.

\section{Consent for publication}

Not applicable.

\section{Competing interests}

The authors declare that they have no competing interests.

\section{Author details}

${ }^{1}$ Gansu Provincial Key Lab of Aridland Crop Science, Lanzhou 730070, Gansu, China. ${ }^{2}$ College of Life Science and Technology, Gansu Agricultural University, Lanzhou 730070, Gansu, China.

Received: 18 August 2021 Accepted: 18 January 2022

Published online: 01 February 2022

\section{References}

1. Yue A, Li A, Mao X, Chang X, Li R, Jing R. Identification and development of a functional marker from 6-SFT-A2 associated with grain weight in wheat. Mol Breeding. 2015;35:63. https://doi.org/10.1007/ s11032-015-0266-9.

2. Lv HH, Yang LH, Kang JG, Wang QB, Wang XW, Fang ZY, et al. Development of InDel markers linked to Fusarium wilt resistance in cabbage. Mol Breeding. 2013;32:961-7. https://doi.org/10.1007/ s11032-013-9925-x.

3. Allen AM, Barker GL, Berry ST, Coghill JA, Gwilliam R, Kirby S, et al. Transcript-specific, single-nucleotide polymorphism discovery and linkage analysis in hexaploid bread wheat (Triticum aestivum L.). Plant Biotechnol J. 2011;9:1086-99. https://doi.org/10.1111/j.1467-7652.2011.00628.x.

4. Jiang Q, Hou J, Hao C, Wang L, Ge H, Dong Y, et al. The wheat (T. aestivum) sucrose synthase 2 gene (TaSus2) active in endosperm development is associated with yield traits. Funct Integr Genomic. 2011;11:49-61. https:// doi.org/10.1007/s10142-010-0188-X.

5. Su Z, Hao C, Wang L, Yuchen D, Zhang X. Identification and development of a functional marker of TaGW2 associated with grain weight in bread wheat (Triticum aestivum L.). Theor Appl Genet. 2011;122:211-23. https:// doi.org/10.1007/s00122-010-1437-Z.

6. Yang Z, Bai Z, Li X, Wang P, Wu Q, Yang L, et al. SNP identification and allelic-specific PCR markers development for TaGW2, a gene linked to wheat kernel weight. Theor Appl Genet. 2012;125:1057-68. https://doi. org/10.1007/s00122-012-1895-6.

7. Qin L, Hao C, Hou J, Wang Y, Li T, Wang L, et al. Homologous haplotypes, expression, genetic effects and geographic distribution of the wheat yield gene TaGW2. BMC Plant Biol. 2014;14:107. https://doi.org/10.1186/ 1471-2229-14-107.

8. MaJun D, Yan J, He Z, Wu L, Xia X. Characterization of a cell wall invertase gene TaCwi - A1 on common wheat chromosome 2A and development of functional markers. Mol Breeding. 2012;29:43-52. https://doi.org/10. 1007/s11032-010-9524-Z.

9. Zhang L, Zhao YL, Gao LF, Zhao G, Zhou RH, Zhang BS, et al. TaCKX6-D1, the ortholog of rice OsCKX2, is associated with grain weight in hexaploid wheat. New Phytol. 2012;195:574-84. https://doi.org/10.1111/j.14698137.2012.04194.X.

10. Guo Y, Sun J, Zhang G, Wang Y, Kong F, Zhao Y, et al. Haplotype, molecular marker and phenotype effects associated with mineral nutrient and grain size traits of TaGS1a in wheat. Field Crop Res. 2013;154:119-25. https:// doi.org/10.1016/j.fcr.2013.07.012.

11. Chang J, Zhang J, Mao X, Li A, Jia J. Polymorphism of TaSAP1-A1 and its association with agronomic traits in wheat. Planta. 2013;237:1495-508. https://doi.org/10.1007/s00425-013-1860-x.

12. Dong L, Wang F, Liu T, Dong Z, Li A, Jing R, et al. Natural variation of TaGASR7-A1 affects grain length in common wheat under multiple cultivation conditions. Mol Breeding. 2014;34:937-47. https://doi.org/10. 1007/s11032-014-0087-2.

13. Zhang Y, Liu J, Xia X, He Z. TaGS-D1, an ortholog of rice OsGS3, is associated with grain weight and grain length in common wheat. Mol Breeding. 2014;34:1097-107. https://doi.org/10.1007/s11032-014-0102-7.

14. Hanif M, Gao F, Liu J, Wen W, Zhang Y, Rasheed A, et al. TaTGW6-A1, an ortholog of rice TGW6, is associated with grain weight and yield in bread wheat. Mol Breeding. 2015;36:1. https://doi.org/10.1007/ s11032-015-0425-z.

15. Hu MJ, Zhang HP, Cao JJ, Zhu XF, Wang SX, Jiang H, et al. Characterization of an IAA-glucose hydrolase gene TaTGW6 associated with grain weight in common wheat (Triticum aestivum L.). Mol Breeding. 2016;36:25. https://doi.org/10.1007/s11032-016-0449-z.

16. Zhang P, He Z, Tian X, Gao F, Xu D, Liu J, et al. Cloning of TaTPP-6AL1 associated with grain weight in bread wheat and development of functional marker. Mol Breeding. 2017;37:78. https://doi.org/10.1007/ s11032-017-0676-y.

17. Halford N, Curtis T, Muttucumaru N, Postles J, Mottram DS. Sugars in crop plants. Ann Appl Biol. 2010;158:1-25. https://doi.org/10.1111/j.1744-7348. 2010.00443.x.

18. Lunn J, Rees T. Apparent equilibrium constant and mass-action ratio for sucrose-phosphate synthase in seeds of Pisum sativum. Biochem. J. 1990;267:739-43. https://doi.org/10.1042/bj2670739.

19. Leloir L, Cardini CE. The biosynthesis of sucrose phosphate. J Biol Chem. 1955;214:157-65. https://doi.org/10.1016/S0021-9258(18)70954-X.

20. Ruan YL. Sucrose metabolism: gateway to diverse carbon use and sugar signaling. Annu Rev Plant Biol. 2014;65:33-67. https://doi.org/10.1146/ annurev-arplant-050213-040251.

21. Castleden CK, Aoki N, Gillespie VJ, MacRae EA, Quick WP, Buchner P, et al. Evolution and function of the sucrose-phosphate synthase gene families in wheat and other grasses. Plant Physiol. 2004;135:1753-64. https://doi. org/10.1104/pp.104.042457.

22. Chen S, Hajirezaei M, Peisker M, Tschiersch H, Sonnewald U, Börnke F. Decreased sucrose-6-phosphate phosphatase level in transgenic tobacco inhibits photosynthesis, alters carbohydrate partitioning, and reduces growth. Planta. 2005;221:479-92. https://doi.org/10.1007/ s00425-004-1458-4.

23. Hawker J, Smith G. Occurrence of sucrose phosphatase in vascular and non-vascular plants. Phytochemistry. 1984;23:245-9. https://doi.org/10. 1016/S0031-9422(00)80311-8.

24. Lunn JE, Ashton AR, Hatch MD, Heldt HW. Purification, molecular cloning, and sequence analysis of sucrose-6F-phosphate phosphohydrolase from plants. Proc Natl Acad Sci U S A. 2000;97:12914-9. https://doi.org/10. 1073/pnas.230430197.

25. Salerno GL, Echeverria E, Pontis HG. Activation of sucrose-phosphate synthase by a protein factor/sucrose-phosphate phosphatase. Cell Mol Biol (Noisy-le-grand). 1996;42:665-72. https://doi.org/10.1111/j.1365-2184. 1996.tb00985.x

26. Echeverria E, Salvucci ME, Gonzalez P, Paris G, Salerno G. Physical and kinetic evidence for an association between sucrose-phosphate synthase and sucrose-phosphate phosphatase. Plant Physiol. 1997;115:223-7. https://doi.org/10.1104/pp.115.1.223.

27. Maloney V, Park JY, Unda F, Mansfield S. Sucrose phosphate synthase and sucrose phosphate phosphatase interact in planta and promote plant growth and biomass accumulation. J Exp Bot. 2015;66:4383-94. https:// doi.org/10.1093/jxb/erv101. 
28. Aravind L, Galperin MY, Koonin EV. The catalytic domain of the P-type ATPase has the haloacid dehalogenase fold. Trends Biochem Sci. 1998;23:127-9. https://doi.org/10.1016/s0968-0004(98)01189-x.

29. Jayashree B, Pradeep R, Kumar A, Gopal B. Correlation between the sucrose synthase protein subfamilies, variations in structure and expression in stress-derived expressed sequence tag datasets. J Proteomics Bioinformatics. 2008;1:408-23. https://doi.org/10.4172/jpb.1000050.

30. Jiang SY, Chi YH, Wang JZ, Zhou JX, Cheng YS, Zhang BL, et al. Sucrose metabolism gene families and their biological functions. Sci Rep. 2015;30:17583. https://doi.org/10.1038/srep17583.

31. Lunn JE. Sucrose-phosphatase gene families in plants. Gene. 2003;303:187-96. https://doi.org/10.1016/s0378-1119(02)01177-0.

32. Lunn JE, MacRae E. New complexities in the synthesis of sucrose. Curr Opin Plant Biol. 2003;6:208-14. https://doi.org/10.1016/s1369-5266(03) 00033-5.

33. Chen S, Hajirezaei MR, Zanor MI, Hornyik C, Debast S, Lacomme C, et al. RNA interference-mediated repression of sucrose-phosphatase in transgenic potato tubers (Solanum tuberosum) strongly affects the hexose-tosucrose ratio upon cold storage with only minor effects on total soluble carbohydrate accumulation. Plant Cell Environ. 2008;31:165-76. https:// doi.org/10.1111/j.1365-3040.2007.01747.X.

34. Dong Y, Zhang Y, Xiao Y, Yan J, Liu J, Wen W, et al. Cloning of TaSST genes associated with water soluble carbohydrate content in bread wheat stems and development of a functional marker. Theor Appl Genet. 2016;129:1061-70. https://doi.org/10.1007/s00122-016-2683-5.

35. Wang D, Liu H, Wang H, Zhang P, Shi C. A novel sucrose transporter gene IbSUT4 involves in plant growth and response to abiotic stress through the ABF-dependent ABA signaling pathway in Sweetpotato. BMC Plant Biol. 2020;20:157. https://doi.org/10.1186/s12870-020-02382-8.

36. Nemati F, Ghanati F, Ahmadi Gavlighi H, Sharifi M. Comparison of sucrose metabolism in wheat seedlings during drought stress and subsequent recovery. Biol Plantarum. 2018;62:595-9. https://doi.org/10.1007/ s10535-018-0792-5.

37. Syamaladevi DP, Jayaraman N, Subramonian N. Structural basis of kinetic variations in Sucrose Phosphate Phosphatase (SPP) of rice and Anabaena unveiled through computational analysis. J Plant Biochem Biot. 2013;23:358-65. https://doi.org/10.1007/s13562-013-0219-5.

38. Lemoine R, La Camera S, Atanassova R, Dédaldéchamp F, Allario T, Pourtau N, et al. Source-to-sink transport of sugar and regulation by environmental factors. Front Plant Sci. 2013;4:272. https://doi.org/10.3389/ fpls.2013.00272.

39. Mathan J, Singh A, Ranjan A. Sucrose transport in response to drought and salt stress involves ABA-mediated induction of OsSWEET13 and OsSWEET15 in rice. Physiol Plant. 2021;171:620-37. https://doi.org/10.1111/ ppl.13210.

40. Du Y, Zhao Q, Chen L, Yao X, Zhang H, Wu J, et al. Effect of drought stress during soybean R2-R6 growth stages on sucrose metabolism in leaf and seed. Int J Mol Sci. 2020;21:618. https://doi.org/10.3390/ijms21020618.

41. Gong X, Liu M, Zhang L, Ruan Y, Ding R, Ji Y, et al. Arabidopsis AtSUC2 and AtSUC4, encoding sucrose transporters, are required for abiotic stress tolerance in an ABA-dependent pathway. Physiol Plantarum. 2014;153:11936. https://doi.org/10.1111/ppl.12225.

42. Zhang YM, Liu ZS, Khan A, Lin Q, Han Y, Mu P, et al. Expression partitioning of homeologs and tandem duplications contribute to salt tolerance in wheat (Triticum aestivum L.). Sci Rep. 2016;6:21476. https://doi.org/10. 1038/srep21476.

43. Mirzaghaderi G, Mason AS. Broadening the bread wheat $D$ genome. TAG. 2019;132(5):1295-307. https://doi.org/10.1007/s00122-019-03299-z.

44. Jia J, Zhao S, Kong X, Li Y, Zhao G, HeW, et al. Aegilops tauschii draft genome sequence reveals a gene repertoire for wheat adaptation. Nature. 2013:496:91-5. https://doi.org/10.1038/nature12028.

45. Zou C, Sun K, Mackaluso J, Seddon A, Jin R, Thomashow M, et al. Cisregulatory code of stress-responsive transcription in Arabidopsis thaliana. Proc Natl Acad Sci U S A. 2011;108:14992-7. https://doi.org/10.1073/pnas. 1103202108.

46. Xue GP, Kooiker M, Drenth J, McIntyre CL. TaMYB13 is a transcriptional activator of fructosyltransferase genes involved in $\beta$-2,6-linked fructan synthesis in wheat. Plant J. 2011;68:857-70. https://doi.org/10.1111/j. 1365-313X.2011.04737.x.

47. Wei H, Zhao H, SUT, Bausewein A, Greiner S, Harms K, et al. Chicory R2R3-MYB transcription factors CiMYB5 and CiMYB3 regulate fructan 1-exohydrolase expression in response to abiotic stress and hormonal cues. J Exp Bot. 2017;68:4323-38. https://doi.org/10.1093/jxb/erx210.

48. Lin S, Singh RK, Moehninsi NDA. R2R3-MYB transcription factors, StmiR858 and sucrose mediate potato flavonol biosynthesis. Hortic Res. 2020;8:25. https://doi.org/10.1038/s41438-021-00463-9.

49. Carbonero P, Iglesias-Fernández R, Vicente-Carbajosa J. The AFL subfamily of B3 transcription factors: evolution and function in angiosperm seeds. J Exp Bot. 2017;68:871-80. https://doi.org/10.1093/jxb/erw458.

50. Wang S, Wong D, Forrest $K$, Allen A, Chao S, Huang BE, et al. Characterization of polyploid wheat genomic diversity using a high-density 90,000 single nucleotide polymorphism array. Plant Biotechnol J. 2014;12:78796. https://doi.org/10.1111/pbi.12183.

51. Allen AM, Winfield MO, Burridge AJ, Downie RC, Benbow HR, Barker $\mathrm{GL}$, et al. Characterization of a wheat breeders' array suitable for highthroughput SNP genotyping of global accessions of hexaploid bread wheat (Triticum aestivum). Plant Biotechnol J. 2017;15:390-401. https:// doi.org/10.1111/pbi.12635.

52. Wang H, Wang S, Chang $X$, Hao C, Sun D, Jing R. Identification of TaPPH$7 \mathrm{~A}$ haplotypes and development of a molecular marker associated with important agronomic traits in common wheat. BMC plant biology. 2019;19:296. https://doi.org/10.1186/s12870-019-1901-0.

53. YEMM EW, WILLIS AJ. The estimation of carbohydrates in plant extracts by anthrone. The Biochem J, 1954;57(3):508-514. https://doi.org/10.1042/ bj0570508.

54. Stein N, Herren GH, Keller B. A new DNA extraction method for highthroughput marker analysis in a large-genome species such as Triticum aestivum. Plant Breeding. 2010;120:354-6. https://doi.org/10.1046/j.14390523.2001.00615.x.

55. Schmittgen TD. Analyzing real-time PCR data by the comparative CT method. Nat Protoc. 2008;3:1101-8. https://doi.org/10.1038/nprot.2008. 73.

56. Guo W, Xin M, Wang Z, Yao Y, Hu Z, Song W, et al. Origin and adaptation to high altitude of Tibetan semi-wild wheat. Nat Commun. 2020;11:5085. https://doi.org/10.1038/s41467-020-18738-5.

57. Hao C, Jiao C, Hou J, Li T, Liu H, Wang YZ, et al. Resequencing of 145 landmark varieties reveals asymmetric sub-genome selection and strong founder genotype effects on wheat breeding in China. Mol Plant. 2020;13:1733-51. https://doi.org/10.1016/j.molp.2020.09.001.

58. Zhou Y, Zhao X, Li Y, Xu J, Bi A, Kang L, et al. Triticum population sequencing provides insights into wheat adaptation. Nat Genet. 2020;52:1412-22. https://doi.org/10.1038/s41588-020-00722-w.

\section{Publisher's Note}

Springer Nature remains neutral with regard to jurisdictional claims in published maps and institutional affiliations.

Ready to submit your research? Choose BMC and benefit from:

- fast, convenient online submission

- thorough peer review by experienced researchers in your field

- rapid publication on acceptance

- support for research data, including large and complex data types

- gold Open Access which fosters wider collaboration and increased citations

- maximum visibility for your research: over $100 \mathrm{M}$ website views per year

At BMC, research is always in progress.

Learn more biomedcentral.com/submissions 